\title{
Philosophiques
}

\section{Guillaume d'Occam et la sémantique des concepts}

Réponses à Elizabeth Karger et à Cyrille Michon

\section{Claude Panaccio}

Volume 21, numéro 2, automne 1994

Les femmes et la société nouvelle

URI : https://id.erudit.org/iderudit/027296ar

DOI : https://doi.org/10.7202/027296ar

Aller au sommaire du numéro

Éditeur(s)

Société de philosophie du Québec

ISSN

0316-2923 (imprimé)

1492-1391 (numérique)

Découvrir la revue

Citer cet article

Panaccio, C. (1994). Guillaume d'Occam et la sémantique des concepts :

réponses à Elizabeth Karger et à Cyrille Michon. Philosophiques, 21(2), 599-605.

https://doi.org/10.7202/027296ar d'utilisation que vous pouvez consulter en ligne.

https://apropos.erudit.org/fr/usagers/politique-dutilisation/ 


\title{
Guillaume d'Occam et la sémantique des concepts Réponses à Élizabeth Karger et a Cyrille Michon
}

\author{
par \\ Claude Panaccio
}

Un auteur peut se considérer choyé d'avoir affaire à des critiques à la fois aussi compétents, aussi perspicaces et aussi sympathiques à son entreprise que ceux auxquels il m'est ici donné de réagir; et je dois avant tout remercier de tout cœur Élizabeth Karger et Cyrille Michon de la lecture attentive et généreuse qu'ils ont faite de mon livre et de la minutie avec laquelle ils se sont employés à en résumer et à en discuter divers aspects. Mes réponses devront, cependant, pour satisfaire aux exigences, du reste bien légitimes, de la revue qui nous accueille, demeurer très succinctes et parfois même un peu sèches. J'essaierai donc de centrer sans détour la discussion sur les principaux points de désaccord qui nous opposent, mes interlocuteurs et moi.

Élizabeth Karger, d'abord, esquisse de la sémantique d'Occam une reconstruction sensiblement différente de la mienne, qui mettrait l'accent sur son rôle dans une logique pure plutôt que dans une théorie nominaliste des concepts mentaux. Rien là que de très légitime, bien sûr. Comme je l'ai expliqué ailleurs ${ }^{\mathrm{I}}$, deux reconstructions divergentes d'un même corpus original peuvent coexister en paix, pour peu que les objectifs des reconstructeurs varient sur tel ou tel point et que leurs propres interventions philosophiques soient clairement identifiables, ce qui est le cas ici. Mais cela n'empêche pas, Karger a raison, qu'on puisse aussi en comparer les mérites respectifs.

Dans le cas présent, la reconstruction qu'elle propose entraîne sur le plan philosophique une complexification - qui me semble indésirable - de la théorie sémantique : multiplication des copules let même des sortes de copules), multiplication des règles de vérité et, surtout, multiplication presque intolérable - des postulats de signification. Mais l'avantage qu'elle réclame pour son approche par rapport à la mienne se situe plutôt au plan de la fidélité à l'entreprise de la Somme de logique. Et il me faut reconnaître, làdessus, que je me suis écarté de mon auteur - d'une manière, du reste, très explicite - en réduisant à quatre dans ma reconstruction les règles établissant les conditions de vérité des propositions simples. La reconstruction kargérienne, cependant, s'en éloigne encore bien davantage en demandant que tous les termes, quelle qu'en soit la complexité de surface, soient « traités comme

I. Cf. C. Panaccio, « De la reconstruction en histoire de la philosophie », dans La philosophie et son histoire, sous la dir. de G. Boss, Zurich, Editions du Grand Midi, 1994, p. 173-195; voir aussi la discussion consacrée à ce texte aux pages 293-312 du même ouvrage. 
des termes simples », au prix d'en admettre « une infinité potentielle » (supra, p. 572). Cela, je dois dire, me parait assez peu compatible avec l'entreprise philosophique d'Occam dans ce qu'elle a de plus déterminant, qui est d'enraciner toute connaissance propositionnelle - et toute logique même - dans le plus petit nombre d'actes simples d'appréhension cognitive. Karger, notamment, doit renoncer à appliquer à l'ordre du langage mental la sémantique pure qu'elle reconstruit ainsi (supra, p. 575). C'est peut-être là une option légitime sur le plan théorique, mais ce n'était pas, je le crains, celle d'Occam, chez qui, au contraire, comme elle dit très bien elle-même, « il est incontestable que sémantique et gnoséologie se rejoignent » (supra, p. 564). Si ma reconstruction se permet par rapport à l'original quelques ajustements locaux, qui me semblent en général justifiés au regard même des exigences théoriques de l'occamisme, celle qu'on lui oppose ici doit renoncer, quant à elle, à faire contribuer la théorie sémantique au projet fondamental d'une gnoséologie à base nominaliste. Cela me semble un écart autrement plus important.

Je ne peux pas croire, en particulier, que Guillaume eût accepté de gaieté de cœur de poser comme entièrement indépendantes les unes des autres la signification d'une expression comme « père de Caïn » et celles de « père » et de « Caïn ». Certes, Karger a raison de noter qu'une telle mise en rapport exigerait, dans une sémantique complète, une explication détaillée de la façon dont la signification des termes complexes dépend de celle des termes simples qui les composent, une entreprise dont Guillaume ne paraît pas avoir très clairement ressenti le besoin. C'est même là l'argument majeur qu'elle invoque à l'appui de son approche. Mais il faut faire ici la part des choses. Les préoccupations logiques d'Occam dans la Summa et la nécessité dans laquelle il se trouvait, étant donné les objectifs de l'ouvrage, d'en venir enfin à cette très longue troisième partie où il expose en détail sa théorie des inférences, lui ont manifestement fait négliger certains volets de la sémantique que son programme théorique aurait requise pour être entièrement complété. La Summa, après tout, qui compte déjà plus de huit cent pages, ne devait être à l'origine qu'un manuel pratique de logique pour débutants ${ }^{2}$ !

Un dernier mot encore, sur une question plus ponctuelle. L'approche proposée par Karger demande, contrairement à la mienne, de ne pas attribuer de supposition personnelle aux prédicats des propositions simples dont le verbe est au passé ou au futur, ce qui, soutient-elle, est plus conforme à la

2. Il est vrai, par ailleurs, que je ne me suis guère employé à retracer les rapports - pourtant très organiques - entre la sémantique mise en place dans les deux premières parties de la Summa et la théorie des inférences de la troisième partie. Mais l'exposé de la difficile logique des inférences n'était pas nécessaire à mon propos, qui était de mettre en valeur la base nominaliste de la sémantique d'Occam et son caractère compositionnel. Sur les rapports entre sémantique et logique chez le venerabilis inceptor, je ne puis faire mieux que renvoyer le lecteur aux articles importants qu'Elizabeth Karger a elle-même consacrés à ces questions, parmi lesquels je voudrais signaler en particulier : « Conséquences et inconséquences de la supposition vide dans la logique d'Ockham », Vivarium, 16, I978, p. 46-55; « Modes of personal supposition : the purpose and usefulness of the doctrine within Ockham's logic », Franciscan Studies, 44, 1984, p. 87-ı6; et « Propositions et syllogismes à l'oblique dans la logique d'Ockham », Archives d'histoire doctrinale et littéraire du Moyen Âge, 60, 1993, p. I47-164. 
doctrine même d'Occam (supra, p. 573). Mais je dois là-dessus marquer mon désaccord. C'est même un point sur lequel Guillaume se montre explicite : «dans la proposition "Socrate était un homme" », écrit-il bel et bien, « le prédicat suppose pour Socrate » (Summa logicae, I, 72, 8I-82). Et il ne peut s'agir ici, de toute évidence, que de suppositio personalis. Il précise, d'ailleurs, dans les lignes qui suivent, que cela vaut pour toutes les propositions au passé ou au futur; et il s'emploie, par la suite, à expliquer de façon générale pour quelles réalités au juste ces prédicats supposent. La position que j'ai adoptée sur ce point est donc bien celle d'Occam. Elle me paraît, en outre, plus simple et plus féconde sur le plan théorique, justement parce qu'elle permet un traitement très unifié des règles de vérité.

Cyrille Michon, de son côté, est nettement plus critique que Karger et que moi à l'endroit d'Occam. D'un certain point de vue, néanmoins, sa perspective rejoint la nôtre. Il voit, lui aussi, dans la pensée du venerabilis inceptor une sorte de paradigme du nominalisme, auquel il est encore fécond, aujourd'hui, de se confronter en philosophie. La différence principale est qu'il espère plutôt le prendre en défaut et faire apparaître ainsi, à travers lui, les apories du nominalisme en général. J'aurai sans doute l'occasion de discuter ailleurs le livre stimulant qu'il vient justement de consacrer à cette entreprise ${ }^{3}$.

Je me réjouis, en tout cas, de constater entre nous un accord très large quant à l'interprétation même des textes d'Occam, une affaire qui est souvent, il faut le dire, assez délicate. Le seul point de divergence que Michon soulève explicitement dans sa recension concerne les rapports entre l'intuition sensible et l'intuition intellectuelle, que Guillaume, contre la plupart de ses prédécesseurs - Thomas D'Aquin en particulier -, tient à distinguer nettement. Il me reproche alors d'avoir décrit « de manière erronée » le processus occamiste de formation du concept (supra, p. 595) lorsque j'ai dit que l'intuition intellective « est causée (au moins partiellement) par l'intuition sensible » (Les mots..., p. II7). « D'après Occam, » affirme au contraire Michon, « la sensation ne cause pas l'intellection [...], elle la précède seulement » (supra, p. 595). Vérification faite, cependant, je dois maintenir ma lecture, qui ne présente, d'ailleurs, dans le cas présent, rien de bien osé, puisque c'est Guillaume luimême qui écrit en toutes lettres dans ses Questions quodlibétales (lesquelles représentent avec la Summa la forme la plus achevée de sa pensée philosophique) : « je dis que la vision sensitive est cause partielle de la vision intellective » (Quodl. I, q. I6, 68-6g). Il est vrai que cela ne vaut que pour la condition humaine d'ici-bas et non plus pour l'âme séparée après la mort, mais la description que je proposais ne devait porter, je n'en ai pas fait mystère, que sur l'ordre naturel et elle est donc, dans ces limites, tout à fait fidèle à la doctrine que j'entendais rapporter : dans le monde qui est le nôtre, la connaissance sensible et la connaissance intellectuelle, pour Occam comme pour Thomas d'Aquin, fonctionnent en série plutôt qu'en parallèle (pour reprendre les expressions suggestives proposées ici par Michon). On ne voit pas que le corps joue chez lui un rôle moindre que chez l'Aquinate. L'un et l'autre, après tout, sont nourris d'Aristote. 
Michon entretient, par ailleurs, des réserves plus sévères quant aux développements - sommaires, il est vrai - que j'ai consacrés ici et là aux prédécesseurs d'Occam. Mais, en fin de compte, la seule objection précise qu'il m'adresse à ce propos repose sur une équivoque. Il me reproche, en effet, « d'attribuer à Thomas d'Aquin [...] une doctrine du concept comme image mentale », alors que, pour cet auteur, rappelle-t-il, « le concept est abstrait à partir des images (phantasmes) » (supra, p. 58I). Mais le différend, ici, tient seulement à ce que Michon veuille réserver le mot français « image » à la traduction de phantasma, alors que je l'employais - malencontreusement, peut-être - pour rendre similitudo ${ }^{4}$ : le concept, pour Thomas comme pour Aristote, est bien un portrait mental de la chose, une similitude dans l'esprit ${ }^{5}$, et je n'ai rien voulu dire d'autre. Cela ne l'empêche pas, pour autant, d'être purement intellectuel, et je ne voulais certainement pas, comme Michon semble le craindre, ramener le concept thomiste à n'être qu'une image d'ordre sensible, un phantasma.

Mais le principal débat, en l'occurrence, est purement philosophique et tourne, il fallait s'y attendre, autour de la question des universaux. Michon trouve que je me suis donné la partie trop belle au chapitre 4 en ne prenant pour adversaires que les platoniciens et les frégéens plutôt que les réalistes dits modérés, « qui refusent de poser des entités universelles séparées des singuliers, mais qui refusent aussi de réduire l'ontologie aux substances et aux qualités singulières » (supra, p. 582). Le véritable rival d'Occam au panthéon des philosophes serait donc, encore une fois, Thomas d' Aquin, le prince des modérés, plutôt que Frege ou Platon, trop radicaux dans leur acceptation des universaux séparés, et c'est avec lui, de préférence, que la discussion devrait se poursuivre aujourd'hui encore. Le thomisme présenterait même, par rapport à l'occamisme, l'insigne avantage d'être plus économique sur le plan de l'ontologie, puisqu'il ne requerrait entre le sujet pensant et la chose singulière extérieure aucun intermédiaire encombrant comme ce concept-signe auquel Guillaume d'Occam accorde, lui, le statut d'une qualité, réellement distincte de l'intellect.

Sur quoi, je rappellerai d'abord, avec Michon, que ce que Guillaume reproche à Thomas d'Aquin, ce n'est pas, avant tout, sa prodigalité ontologique, mais l'incohérence de sa doctrine. Bien au-dessus de la maxime d'économie, la règle d'or en philosophie, pour Occam, est le principe de noncontradiction. Or c'est cette contrainte inéluctable que doit violer le réalisme modéré d'un Thomas d'Aquin pour éviter le platonisme. Il est bien vrai que le

4. Je suivais en cela la traduction du Peri hermeneias d'Aristote (16a8) par Tricot, lequel utilise justement « image » pour rendre le mot grec homoiôma, devenu similitudo en latin, chez Boèce et Thomas d'Aquin notamment.

5. Les textes abondent à ce sujet. Voir en particulier : Quaestiones de potentia Dei, q. 8, art. I : « le verbe qui naît de l'intellect est une similitude de la chose intelligée » (N.B. : le « verbe »chez Thomas d'Aquin est la même chose que le concept). Voir aussi entre autres le Commentaire de l'Evangile de Saint-Jean, lect. I, I, parg. 25, et le Commentaire du Peri hermeneias, lect. 2, parg. Ig. La même chose vaut d'ailleurs pour la species intelligibilis, que Thomas distingue pourtant du concept (voir, par exemple, Somme de théologie, I, 85, art. 2, ad I : « L'objet connu est dans l'intelligence connaissante par sa ressemblance »). Thomas, du reste, distingue très bien en général entre la similitudo dans les sens (le phantasma) et la similitudo dans l'intellect. 
Docteur angélique, comme le venerabilis inceptor après lui, est en quête d'une théorie de la connaissance qui ne l'oblige pas à admettre la position des universaux comme entités séparées. Mais le problème est qu'il n'y parvient pas, du moins pas sans se contredire. La clé de sa solution, comme Michon le rappelle, est l'identification intentionnelle du concept à l'objet : « le concept n'est pas une autre chose que son objet, il est cet objet dans un être intentionnel » (supra, p. 594); et c'est cette identification, justement, qui permettrait d'éviter l'intervention d'un intermédiaire entre le sujet connaissant et les choses connues. Mais Thomas, du même souffle, doit aussi reconnaître, comme tout le monde, que le concept n'est pas uraiment identique à la chose connue ${ }^{6}$. Comment pourrait-il faire autrement, d'ailleurs, puisque la chose extérieure, à ses yeux mêmes, est matérielle et singulière tandis que le concept n'est ni l'un ni l'autre? Sous peine de contradiction, il faut renoncer à dire que l'identité intentionnelle est une identité au sens fort; et l'assimilation intentionnelle, dès lors, n'explique plus rien. D'une part, elle n'empêche pas la position d'entités intermédiaires, que le thomisme multiplie même puisqu'il distingue le concept non seulement de la chose extérieure (comme on vient de le voir) et du sujet connaissant (qui est, en effet, pour Thomas d'Aquin, bien autre chose qu'un concept), mais aussi de la species intelligibilis comme de l'acte intellectif, tous les deux posés par Thomas comme autant de qualités mentales distinctes ${ }^{7}$. Et d'autre part, l'identité intentionnelle ne permet pas non plus de faire l'économie de l'universel hors de l'esprit : le concept étant fondamentalement un universel, s'il est identique, ne fût-ce qu'intentionnellement, à quelque chose hors de l'esprit (comme la forme?), cette chose-là doit bien être elle-même un universel. Le réalisme modéré n'évite la contradiction ou la vacuité qu'en retombant dans une variante ou une autre de platonisme.

C'est un point sur lequel Guillaume d'Occam a souvent insisté. Si je n'ai pas jugé bon de reprendre toute cette discussion au chapitre 4 de mon livre, c'est que le propos en était seulement de faire dialoguer Occam avec Nelson Goodman. Le rappel sommaire de certaines positions réalistes et de leurs difficultés dans la première section du chapitre n'avait d'autre but que de fournir un contexte à cette confrontation en rappelant les principales positions en présence. Il m'apparaît encore que le débat le plus pertinent à cet égard était bien celui du nominalisme avec le platonisme de type frégéen ${ }^{8}$.

6. Voir, par exemple, les Quaestiones de potentia Dei, q. 8, art. I, où Thomas rappelle, avec beaucoup de bon sens, que le concept « diffère de la chose intelligée, parce que celle-ci est extérieure à l'intellect, alors que le concept, lui, n'est nulle part ailleurs que dans l'intellect ».

7. L'article des Quaestiones de potentia Dei cité à la note précédente est à ce sujet tout à fait explicite. Je me permets, pour plus de détails, de renvoyer là-dessus le lecteur à mon texte « From mental word to mental language » dans Philosophical Topics, 20, 2, 1992, p. 125-147.

8. Il y a, bien sûr, d'autres positions réalistes aujourd'hui qui valent d'être discutées, comme celle de l'australien D.M. Armstrong dans son livre remarquable, A Theory of Universals (Cambridge, Cambridge University Press, 1978, 2 vol.), sur laquelle Michon attire l'attention. Mais Armstrong reconnaît lui-même que la plupart de ses arguments antinominalistes ne porteraient pas contre un nominalisme qui admettrait les possibilia, ce qui est précisément le cas de l'occamisme comme je l'interprète. 
La stratégie occamiste, en tout cas, repose essentiellement sur la caractérisation du concept mental comme signe et c'est sur ce point justement que Michon émet ses réserves les plus graves. Rappelant que la Somme delogique distingue dès le premier chapitre entre deux sens du mot signum, il en conclut que le concept, chez Occam, « n'est pas un signe au sens où tous les autres signes sont des signes » et que la notion clé du concept-signe est, par conséquent, « parfaitement obscure » (supra, p. 592). Mais il y a ici quiproquo. La deuxième définition occamiste de signum - la seule qui nous intéresse en l'occurrence - ne doit pas valoir seulement pour les concepts, mais également pour tous les signes d'ordre linguistique, fussent-ils conventionnels. Le signum, en ce sens, est « cela qui fait venir quelque chose à la connaissance et qui est destiné (natum est) à supposer pour cette chose, ou bien à être ajouté à ce qui suppose dans une proposition, comme c'est le cas des syncatégorèmes... »9. Le concept est donc un signe exactement au même sens que le sont les mots oraux ou écrits. La distinction des deux sens de signum n'a pour but, comme l'explique Occam lui-même, que de calmer les coupeurs de cheveux en quatre (propter protervos, dit-il) en écartant du débat l'autre définition du signe comme cela qui renvoie à quelque chose de déjà connu. Ce qui importe pour le franciscain dans l'inscription du concept sous la catégorie des signes, c'est justement d'en mettre en évidence les affinités essentielles avec les termes linguistiques - ceux qui peuvent figurer dans des phrases - et d'ouvrir ainsi la voie à l'utilisation de la terminologie sémantique (significatio, suppositio, connotatio, etc.) pour l'analyse fine des processus cognitifs. L'intérêt de la manœuvre doit se juger à ceci qu'elle rend possible, pour la première fois dans l'histoire des théories de la connaissance, une analyse compositionnelle détaillée de la pensée humaine ${ }^{\text {Io }}$.

La question cruciale, évidemment, est de savoir si cette analyse peut être menée à terme sans engagement ontologique indésirable pour un nominaliste. Michon en doute et pense que ma reconstruction de l'occamisme est commise à l'admission des ensembles (supra, p. 579) et à celle des modes d'être ou des relations (supra, p. 586). Il ne discute pas, cependant, les deux dispositifs théoriques par lesquels j'avais essayé, justement, d'éviter ces écueils séculaires : l'introduction, d'une part, d'une notion restreinte d'appartenance à un ensemble par le biais d'une définition constructive qui ne renvoie à rien d'autre qu'à des entités singulières - y compris des signes ${ }^{\text {II }} \rightarrow$, et le recours, d'autre part, à cette généralisation de la connotation occamiste quej'ai appelée

9. Il est possible que la confusion, ici, provienne de l'expression « natum est » que Michon, reprenant la traduction de Joël Biard, rend par « est destiné par nature », ce qui le porte peutêtre à croire que la définition en question n'est pas censée s'appliquer aux signes conventionnels, comme les mots oraux ou écrits. Mais le contexte montre qu'il n'en est rien et Biard le signale d'ailleurs en note (Guillaume d'Ockham, Somme de logique. Première partie, Mauvezin, T.E.R., $2^{\mathrm{e}}$ éd., I993. p. 7, n. 6).

Io. J'aurai l'occasion de développer cette dernière thèse en long et en large dans un ouvrage en préparation sur la formation de l'idée de langage mental à partir de Platon et d'Aristote jusqu'à Guillaume d'Occam.

II. Voir Les mots..., p. $44^{-} 5$. 
la fonction associative ${ }^{12}$. Il est vrai que l'une et l'autre idées restent trop peu développées dans mon livre et je ne saurais prétendre avoir ainsi réglé sans appel des questions qui sont parmi les plus délicates de toute la philosophie. Mais en l'absence d'objections ou de questions précises à leur sujet, je ne saurais, dans les limites du présent échange, en pousser plus avant la discussion. Je me contenterai seulement de désavouer - et de bonne grâce - la « tranquille assurance » que Michon m'attribue à ce propos : je suis loin d'être « sûr » que le nominalisme soit vrai. Mais en dépit des nombreuses réfutations qui ont prétendu en venir à bout au cours des siècles (le nôtre en particulier), son programme mérite encore d'être exploré avec sérieux. Quant à moi, il me suffit amplement d'avoir pu contribuer par mon livre à maintenir vivantes dans le monde francophone des préoccupations et des doctrines que j'estime importantes. La qualité des réactions comme celles qu'expriment ici Élizabeth Karger et Cyrille Michon me comble tout à fait.

\section{Département de philosophie}

Université du Québec à Trois-Rivières

12. Id., p. 48-9; j'ai aussi voulu suggèrer que ces notions de fonction associative et de connotation ne peuvent contribuer efficacement à la simplification de l'ontologie qu'en ètant couplées à une sémantique des cas à la Fillmore (id., p. 240-7). 